\title{
De novo assembly of middle-sized genome using MinION and Illumina sequencers
}

\author{
Ryuhei Minei, Ryo Hoshina and Atsushi Ogura * (D)
}

\begin{abstract}
Background: The plastid acquisition by secondary endosymbiosis is a driving force for the algal evolution, and the comparative genomics was required to examine the genomic change of symbiont. Therefore, we established a pipeline of a de novo assembly of middle-sized genomes at a low cost and with high quality using long and short reads.

Results: We sequenced symbiotic algae Chlorella variabilis using Oxfofrd Nanopore MinlON as the long-read sequencer and Illumina HiSeq 4000 as the short-read sequencer and then assembled the genomes under various conditions. Subsequently, we evaluated these assemblies by the gene model quality and RNA-seq mapping rate. We found that long-read only assembly could not be suitable for the comparative genomics studies, but with short reads, we could obtain the acceptable assembly. On the basis of this result, we established the pipeline of de novo assembly for middlesized algal genome using MinlON.
\end{abstract}

Conclusions: The genomic change during the early stages of plastid acquisition can now be revealed by sequencing and comparing many algal genomes. Moreover, this pipeline offers a solution for the assembly of various middle-sized eukaryotic genomes with high-quality and ease.

Keywords: Plastid acquisition, Secondary endosymbiosis, Chlorella variabilis, Genome assembly pipeline, Hybrid assembly, Gene model quality, RNA-seq mapping rate

\section{Background}

Evolutionary research has revealed divergence in photosynthetic eukaryotes with plastid acquisition by secondary endosymbiosis as the driving force [1-3]. This phenomenon has occurred many times over the course of evolution. Plastid acquisition by secondary endosymbiosis consists of four stages: In the first stage, host organisms prey on algae, and undigested algae temporarily become symbionts. In the second stage, the temporary symbionts become persistent symbionts. In the third stage, horizontal gene transfer from the symbiont to the host nucleus occurs. In the final stage, the nucleus of the symbiont disappears, resulting in the establishment of a plastid [4]. Curtis et al. [5] previously studied the third stage of plastid acquisition by genome and transcriptome analysis of cryptophytes and chlorarachniophytes. However, few studies have focused on the early stages of plastid acquisition.

\footnotetext{
* Correspondence: aogu@whelix.info

Department of BioScience, Nagahama Institute of Bio-Science and

Technology, Tamura 1266, Nagahama, Shiga 526-0829, Japan
}

The symbiotic relationship between the ciliate Paramecium bursaria and the symbiotic green algae Chlorella variabilis is considered a model of the second stage of plastid acquisition [6]. Blanc et al. [7] sequenced the genome of $C$. variabilis to study the genetic basis of symbiosis. However, as genome data were not available for symbionts other than C. variabilis and closely related species, it was not possible to compare genomes to clarify the genomic changes associated with becoming a symbiont. To shed light on the genomic change of the early stages of plastid acquisition, it is necessary to sequence the genomes of symbionts and free-living species other than C. variabilis; there are many species of symbiotic green algae, and the host organisms have diversified to Ciliates (Alveolata) as well as Amoebozoa, Heliozoa, and other protists [8]. Thus, we must examine genomic changes in these symbionts and the transcriptomic interactions of hosts and symbionts. High-quality assemblies of multiple isolates of both free-living and symbiotic Chlorella are needed to address the second stage of plastid acquisition. For this purpose, it is necessary to assemble many middle-sized (30 60 megabase 
pairs) algal genomes for detailed analysis, which requires a low cost and high-quality method [9-11].

For high-quality genome assembly that reflects gene content and genome structure, long-read sequencers are preferred to short-read sequencers. Read lengths from short-read sequencers are 50-400 bp, resulting in highly fragmented genome assemblies. By contrast, long-read sequencers can sequence long-repeated regions and copy number variations, providing reads up to hundreds of kilobases (kbp) in length [12]. However, long-read sequencers such as PacBio RS2 and Sequel have high costs [13]. They are not suitable for sequencing many green algae genomes. Therefore, we chose the Oxford Nanopore MinION long-read sequencer, which has been distributed since 2014, for this work. MinION has five advantages: the low cost of sequencing, ultra-long reads up to $200 \mathrm{kbp}$, portability, real-time analysis, and direct molecule analysis [12]. For these reasons, MinION has the potential to revolutionize genomic research. However, there are few reports validating de novo assembly of middle-sized genomes using MinION.

Here, we aimed to establish a pipeline of de novo assembly for middle-sized genomes at a low cost and with high-quality using MinION for multiple green algal species. We sequenced $C$. variabilis using MinION as the long-read sequencer and Illumina HiSeq 4000 as the short-read sequencer and then assembled the genomes under various conditions (Fig. 1). Subsequently, we evaluated these assemblies by assessing whether contigs were successfully connected to each other (assembly quality). We also compared these assemblies to a high-quality reference previously assembled by Blanc et al. [7] using Sanger sequencing. Lastly, we examined if these assemblies could be used for actual comparative genomics using the gene model quality and RNA-seq mapping rate.

\section{Results and discussion}

\section{Comparison of assemblies under various conditions}

Sequencing $C$. variabilis using MinION and HiSeq generated 78X depth short reads and 56X depth of 578,473 bp long reads. In previous studies, the Escherichia coli genome was assembled using 20X MinION reads [14], and the Saccharomyces cerevisiae genome was assembled using 30X MinION reads [15]. A depth of $56 \mathrm{X}$ is considered a sufficient amount of sequence reads to assemble the chlorella genome. Regarding sequenced raw reads from MinION, we obtained relatively long reads, with an average length of $4.45 \mathrm{kbp}$, maximum read length of $766,552 \mathrm{bp}$, and sequencing accuracy of $87.4 \%$ (Additional file 1: Figure S1 and Additional file 2: Table S1).

Using these reads, we assembled genomes under various conditions. We assembled genome using only short reads by SPAdes [16] assembler (short-read assembly). Using only long reads, we assembled genomes using three assemblers, namely, ABrujin [17] (ABrujin-assembly), Miniasm [18] (Miniasm-assembly), and Canu [19] (Canu-assembly), which are commonly used in published papers. In addition, we tested the hybrid genome

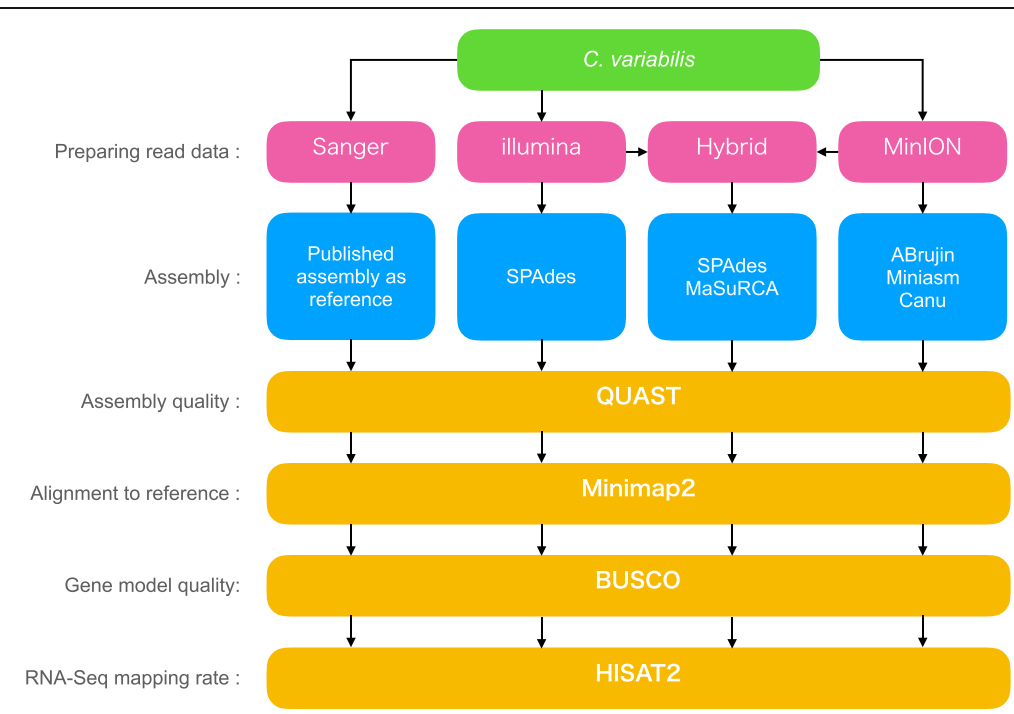

Fig. 1 Overview of the workflow for comparing assemblies under various conditions. First, genomic DNA extraction of $C$. variabilis is shown in green. Sanger, Illumina, and MinION indicates sequence reads data derived from these sequencers in the second step (pink). Hybrid indicates mixed data of Illumina and MinION. In the third step (blue), genome assembly under various conditions was performed, and each term shows the name of assembler software. Assembly using reads derived from Sanger sequencer was taken from previously published data [7]. The next steps (yellow) are evaluation of assemblies, each term shows the name of software. The alignment to reference indicates result of aligning each assembly to reference 
assembly of long and short reads using the SPAdes [14] (SPAdes-hybrid-assembly) and MaSuRCA assemblers [20] (MaSuRCA-assembly). We evaluated assemblies by assembly quality, the coverage, and percent sequence identity by aligning each assembly to the reference. The quality of the reference assembly using Sanger sequencing was relatively good: the number of contigs was small, and the largest contig and N50 were long, as it was assembled using relatively long reads (400 $900 \mathrm{bp}$ ). However, this reference has two problems. First, the number of Ns per $100 \mathrm{kbp}$ was large (8547) and inserted upon scaffolding contigs, preventing precise gene model assumptions. Second, most of the contigs were very small even though the largest contig and N50 were long (Table 1).
Comparing the short assembly with the reference, the number of contigs was large and the largest contig and N50 were short, indicating difficulty in scaffolding from short reads only. Additionally, the short-read-assembly had 14,208,652 bp that did not align to reference, suggesting that the assembly using only short reads contains many inaccurate sequences (Table 1). In assemblies using only long reads, ABrujin-assembly, and Miniasmassembly covered almost the whole genome of $C$. variabilis as the coverage rates exceeded $90 \%$. ABrujin-assembly had the highest assembly quality among assemblies using only long reads. In particular, ABrujin-assembly was able to assemble the reads into a small number of contigs than the reference. Although the largest contig and N50 in ABrujin-assembly were shorter than in the

Table 1 Summary of evaluation for assembled genomes of C. variabilis under various conditions using various indexes

\begin{tabular}{|c|c|c|c|c|c|c|c|c|}
\hline & \multirow[t]{2}{*}{ Reference } & \multirow[t]{2}{*}{ Short-read } & \multicolumn{4}{|l|}{ Long } & \multicolumn{2}{|l|}{ Hybrid } \\
\hline & & & ABrujin & Abrujin-polished & Miniasm & Canu & SPAdes-hybrid & MaSuRCA \\
\hline \multicolumn{9}{|l|}{ Assembly quality } \\
\hline \# contigs (> = $0 \mathrm{bp}$ ) & 414 & 13,015 & 259 & 170 & 492 & 2400 & 10,635 & 302 \\
\hline \# contigs ( $>=1000$ bp) & 414 & 1870 & 259 & 170 & 492 & 2399 & 1079 & 302 \\
\hline \# contigs ( $>=5000$ bp) & 134 & 1171 & 259 & 170 & 484 & 1909 & 772 & 241 \\
\hline \# contigs ( $>=10,000 \mathrm{bp}$ ) & 82 & 950 & 259 & 170 & 438 & 1158 & 664 & 196 \\
\hline \# contigs ( $>=25,000 \mathrm{bp}$ ) & 55 & 642 & 259 & 170 & 335 & 141 & 511 & 150 \\
\hline \# contigs ( $>=50,000 \mathrm{bp}$ ) & 44 & 348 & 225 & 164 & 243 & 18 & 359 & 134 \\
\hline Total length (> =0 bp) & $46,159,515$ & $58,108,416$ & $44,173,773$ & $45,397,519$ & $42,468,310$ & $27,800,588$ & $58,637,084$ & $46,674,734$ \\
\hline Total length $(>=1000 \mathrm{bp})$ & $46,159,515$ & $56,312,404$ & $44,173,773$ & $45,397,519$ & $42,468,310$ & $27,799,589$ & $57,237,973$ & $46,674,734$ \\
\hline Total length (> = $5000 \mathrm{bp}$ ) & $45,602,804$ & $54,680,763$ & $44,173,773$ & $45,397,519$ & $42,437,055$ & $26,338,534$ & $56,523,201$ & $46,494,724$ \\
\hline Total length $(>=10,000 \mathrm{bp})$ & $45,222,671$ & $53,103,981$ & $44,173,773$ & $45,397,519$ & $42,054,109$ & $20,603,401$ & $55,758,312$ & $46,162,375$ \\
\hline Total length $(>=25,000 \mathrm{bp})$ & $44,846,071$ & $48,059,147$ & $44,173,773$ & $45,397,519$ & $40,395,860$ & $5,591,416$ & $53,252,484$ & $45,478,395$ \\
\hline Total length $(>=50,000 \mathrm{bp})$ & $44,435,177$ & $37,767,367$ & $42,798,957$ & $45,170,192$ & $36,950,227$ & $1,766,192$ & $47,753,927$ & $44,916,352$ \\
\hline \# contigs & 414 & 2540 & 259 & 170 & 492 & 2400 & 1479 & 302 \\
\hline Largest contig & $3,119,887$ & 765,833 & $1,514,322$ & $1,157,783$ & 685,202 & 327,336 & 770,020 & $2,552,940$ \\
\hline Total length & $46,159,515$ & $56,771,972$ & $44,173,773$ & $45,397,519$ & $42,468,310$ & $27,800,588$ & $57,502,328$ & $46,674,734$ \\
\hline GC (\%) & 67.1 & 67.9 & 64.4 & 65.8 & 64.8 & 62.2 & 67.8 & 67.1 \\
\hline N50 & $1,469,606$ & 77,546 & 250,313 & 376,772 & 161,216 & 14,421 & 130,737 & 501,441 \\
\hline N75 & 953,202 & 36,956 & 137,022 & 228,146 & 84,461 & 9866 & 66,441 & 250,951 \\
\hline L50 & 12 & 195 & 54 & 35 & 77 & 599 & 127 & 23 \\
\hline L75 & 21 & 462 & 113 & 75 & 166 & 1183 & 279 & 55 \\
\hline \# N's per $100 \mathrm{kbp}$ & 8547 & 27 & 0 & 0 & 0 & 0 & 139 & 13 \\
\hline \multicolumn{9}{|l|}{ Alignment to reference } \\
\hline Coverage & - & $92.21 \%$ & $94.68 \%$ & $99.73 \%$ & $90.23 \%$ & $26.09 \%$ & $93.76 \%$ & $100.77 \%$ \\
\hline Identity & - & $89.19 \%$ & $21.16 \%$ & $69.82 \%$ & $14.69 \%$ & $2.56 \%$ & $89.96 \%$ & $97.23 \%$ \\
\hline \multicolumn{9}{|l|}{ Gene model quality } \\
\hline ECR & $82.90 \%$ & $86.50 \%$ & $1.30 \%$ & $10.90 \%$ & $0.00 \%$ & $0.00 \%$ & $86.80 \%$ & $88.50 \%$ \\
\hline RNA-seq Mapping Rate & $90.32 \%$ & $95.81 \%$ & $23.08 \%$ & $56.81 \%$ & $18.68 \%$ & $5.74 \%$ & $96.25 \%$ & $95.06 \%$ \\
\hline
\end{tabular}

Statistics of assembly quality are based on contigs of size > = 500 bp, unless otherwise noted (e.g., "\# contigs (>=0 bp)" and "Total length ( $>=0$ bp)" include all contigs). Identity indicats the percent sequence identity to the reference 
reference, the number of contigs over $50 \mathrm{kbp}$ (225) was larger and each contig was longer on average, suggesting that assembly using long reads is suitable for analysis of clustered genes and synteny. Conversely, the Canu-assembly did not cover the whole genome of $C$. variabilis and the total size of the genome $(27,800,588 \mathrm{bp})$ was much smaller than of the reference (about $46 \mathrm{Mbp}$ ), and had remarkably lower indices of assembly quality than those for the other assemblies, suggesting that this assembler is not suitable for assembling the $C$. variabilis genome using long reads only (Table 1 ). Regarding hybrid assembly, SPAdes-hybrid-assembly and MaSuRCA-assembly had a higher coverage and percent sequence identity to the reference than the short-read-assembly. However, SPAdes-hybrid-assembly gave the assembly quality as low as that of short-read-assembly, since scaffolding using long reads was insufficient. On the other hand, the MaSuRCA-assembly resulted in a smaller number of contigs than the reference, a larger contig and N50 than the ABrujin-assembly, and the highest assembly quality. The values of the MaSuRCA-assembly were the highest among our results. In summary, the percent sequence identity to the reference using long reads was lower than that using short-read-assembly or assemblies using only short reads, as the sequencing accuracy of MinION was low. By contrast, hybrid assembly showed improved results as the accuracy of short reads could correct the sequences from MinION.

We assumed that a high-quality assembly could be obtained using only long reads from MinION if the sequencing errors were improved, as the assembly quality and coverage of the ABrujin-assembly were high. Consequently, we polished ABrujin-assembly (ABrujin-polishe$\mathrm{d}$-assembly), which is the highest evaluated assembly among the long-read assemblies, using Racon [21] and Nanopolish [22]. As a result, the percent sequence identity to the reference for the ABrujin-assembly improved from 22.16 to $69.82 \%$ (Table 1). However, the improved percent sequence identity was still lower than that for short-read-assembly.

\section{Gene model quality}

Precise prediction of genes is essential for comparative genomics to reveal the common process in the second stage of plastid acquisition related to gene duplication, gene loss, and horizontal gene transfer. Therefore, we evaluated the precision of gene recovered in the assemblies constructed above by the common eukaryotic genes conservation rate (ECR) using BUSCO [23]. ECR is defined as the proportion of 303 common eukaryotic single-copy-orthologs (core genes) recovered in the genome assemblies in comparison [23]. The ECR of the reference, short-read-assembly, ABrujin-assembly, ABrujin-polished-assembly, Miniasm-assembly, Canu- assembly, SPAdes-hybrid-assembly, and MaSuRCA-assembly were $82.90 \%, 88.50 \%, 1.30 \%, 10.90 \%, 0.00 \%$, $0.00 \%, 86.80 \%$, and $88.50 \%$, respectively (Table 1 ). The reason why the ECR of the short-read-assembly and SPAdes-hybrid-assembly was higher than that of reference is that these assemblies had 19 duplicated core genes (Fig. 2a). These duplications are artifacts of low assembly quality. The ECRs were quite low for assemblies using only long reads, as the sequencing accuracy of MinION is low. Though its percent sequence identity was remarkably improved by polishing, the ECR of ABrujin-polished-assembly was still extremely low. This indicates that the ABrujin-polished-assembly is not useful for actual genome analysis. The ECR of the MaSuRCA-assembly was the highest of all assemblies including the reference, and core gene duplication was not observed.

Next, we performed whole-gene model prediction using the short-read-assembly, ABrujin-polished-assembly and MaSuRCA-assembly, and evaluated the number and average length of the gene model to compare these gene models to the reference gene model previously proposed [7]. In the gene model for the short-read-assembly, the number of genes was more than double that for the reference gene model, and the average length of genes was different from that for the reference gene model. In the gene model for the ABrujin-polished-assembly, the average length of genes was dramatically lower than for the other models, indicating that the ABrujin-polished-assembly model is not suitable for genome analysis because start and stop codons were incorrectly introduced in the middle of the coding sequence in this model.

\section{Evaluation of RNA-seq mapping rate}

Gene expression analysis can provide essential clues to help us understand how hosts and symbiont live together at the second stage of plastid acquisition. This analyses should be processed by mapping RNA-seq to the assembled genome, not by de novo assembly of RNA-seq, as it is crucial to identify the origin of the highly conserved genes. We evaluated whether reliable gene expression analysis could be performed by calculating the RNA-seq mapping rate against these assemblies using HISAT2 [24]. The RNA-seq mapping rates for the reference, the short-read-assembly, ABrujin-assembly, ABrujin-polished-assembly, Miniasm-assembly, Canu-assembly, SPAdes-hybrid-assembly, and MaSuRCA-assembly were $90.32 \%, 95.81 \%, 23.08 \%, 56.81 \%, 18.68 \%, 5.74 \%$, $96.25 \%$, and $95.06 \%$ (Table 1). The reason why the RNA-seq mapping rates for the short-read-assembly and hybrid assemblies were higher than those for the reference assembly is because the number of Ns per $100 \mathrm{kbp}$ of reference was large and the same sequencing error profile of HiSeq gives raised sequence identity. For the assemblies 


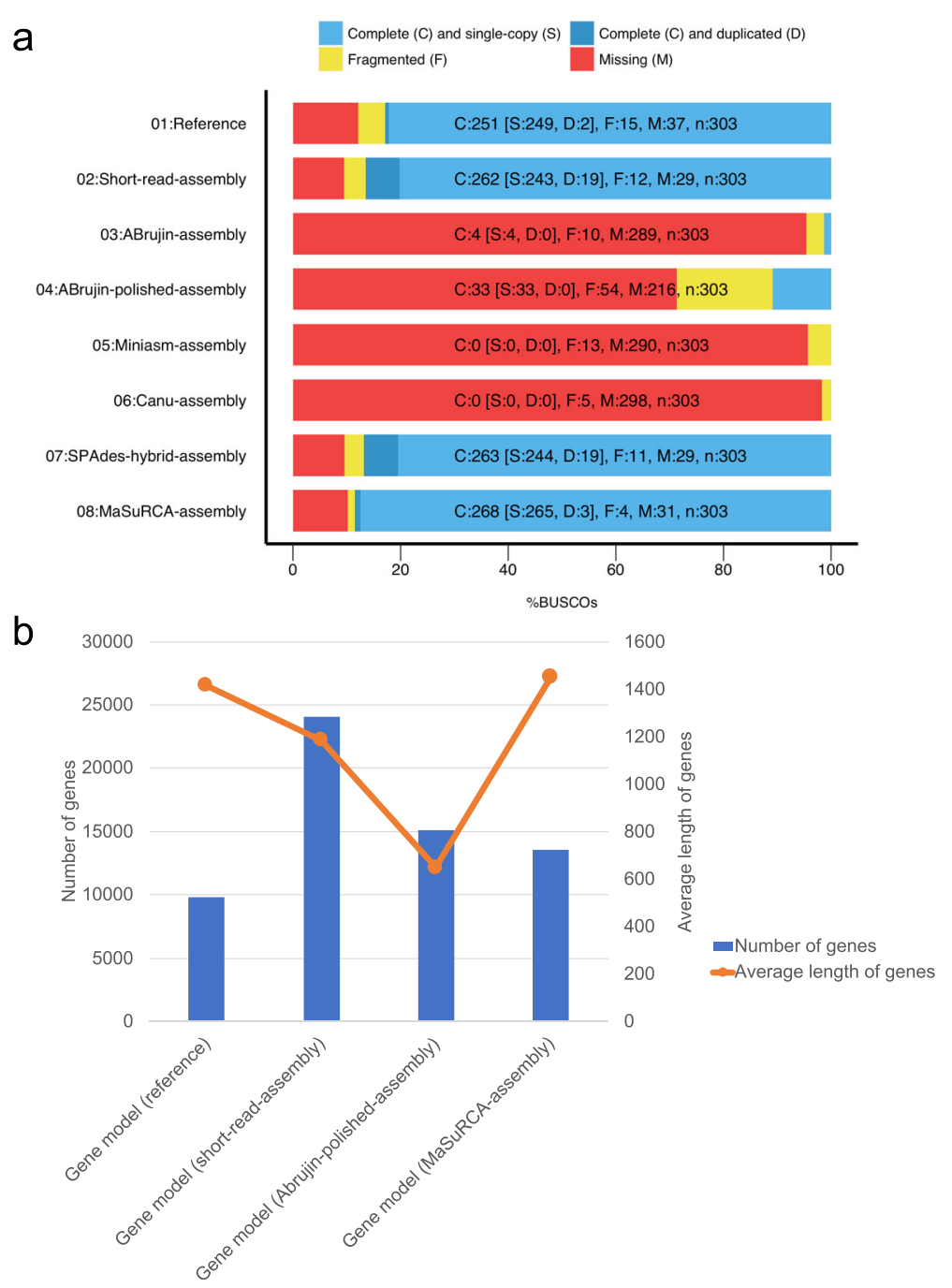

Fig. 2 Evaluation of assemblies using gene model quality. a Assessment of ECR on eight assemblies using BUSCO. The x-axis represents of ECR patterns. $\mathbf{b}$ Evaluation of estimated four gene models of four assemblies using average length of genes (orange line) and the number of genes (blue bar).

using only long reads, the RNA-seq mapping rate was quite low, as the sequencing accuracy of MinION is low. In addition, the RNA-seq mapping rate of the ABrujinpolished-assembly, whose percent sequence identity to the reference was low even after polishing, suggests that this assembly cannot be sufficiently improved by polishing and is not suitable for gene expression analysis.

We also investigated how much the gene expression levels of these assemblies differed from each other, which reflects the quality of the short-read-assembly, ABrujin-polished-assembly, and MaSuRCA-assembly. To obtain the same genes from these assemblies, we predicted orthologs and extracted 5343 single-copy orthologs suitable for comparing gene expression levels without the effects of paralogous genes. We then calculated the transcripts per kilobase million (TPM) [25] of these single-copy orthologs in each assembly. Finally, we evaluated the correlations between gene expression levels using scatterplots and correlation testing. The correlation coefficient between the short-read-assembly and MaSuRCA-assembly was the highest (0.9997) among all combinations, followed by the reference and MaSuRCA-assembly (0.9832) and the reference and short-read-assembly (0.9831) (Fig. 3). By contrast, the correlation coefficients of the ABrujin-polished-assembly using only long leads were the lowest of all assemblies (vs. reference 0.9178, short-read-assembly 0.9209, and MaSuRCA-assembly 0.9210) (Fig. 3). Differences in the correlation coefficients occurred for the same reason as in the mapping rates. The assembly using only long reads was not suitable for gene expression analysis as TPM greatly varied. 


\section{Reference}

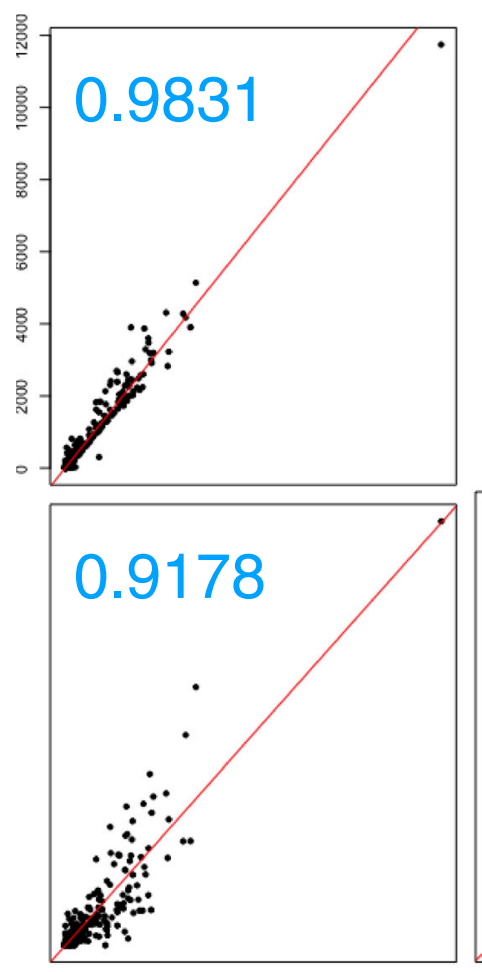

\section{Short}
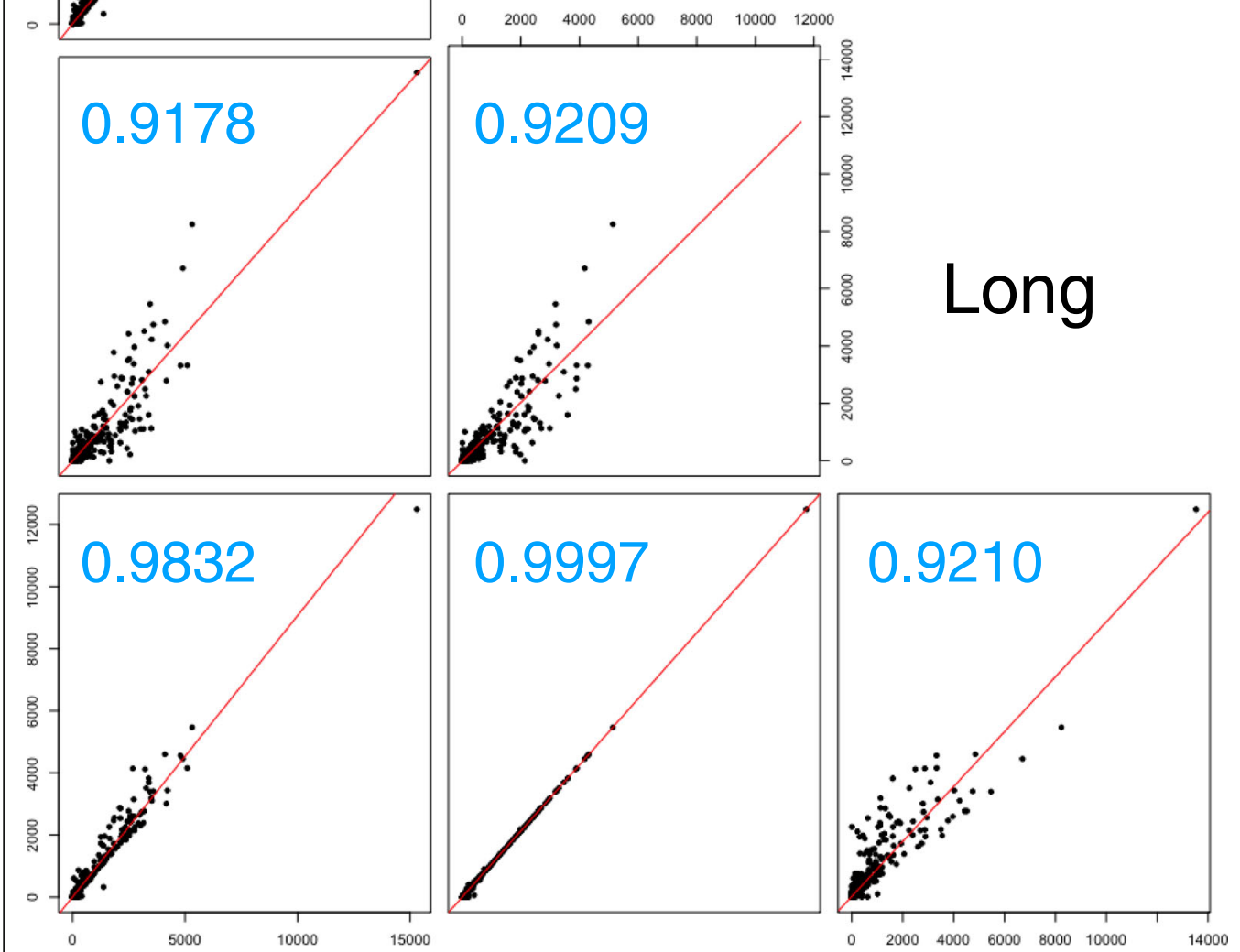

\section{Hybrid}

Fig. 3 Correlation analysis of the gene expression level among four assemblies. Scatter plots demonstrates the TPM of single-copy-orthologs between all pair-wise assemblies. Each dot corresponds to a single gene. The red lines show regression line. The blue digits on the top right indicate the correlation coefficient value between each assembly. The Reference, Short, Long, and Hybrid show reference assembly previously published, short-read-assembly, ABrujin-polished-assembly, and MaSuRCA-assembly, respectively

Establishment of a de novo assembly pipeline for middlesized genomes using MinION

The quality of assembly using only long reads was lower than that for other assemblies mainly because of the low sequencing accuracy, which is a restriction of the current nanopore technology. Consequently, it was not suitable for comparative genomics and transcriptomics as it reduces the precision of gene prediction and the
RNA-seq mapping rate. Furthermore, these indices were not improved by polishing, indicating that assembling a high-quality genome of $C$. variabilis with only $56 \mathrm{X}$ MinION reads data is difficult. However, this problem was remedied by hybrid assembly using short reads; we established a pipeline for de novo assembly of middlesized genomes using MinION long reads with Illumina short reads using the MaSuRCA-assembly. 
As there are many target species of symbiotic algae for comparative genomic research, it is worthwhile to identify a better sequencing strategy with lower sequence read coverages. In addition, preparing massive and high-quality genomic DNA for these species that is long but fragmented enough to sequence using MinION is difficult with respect to culturing [26], and finding a smaller amount of input genomic DNA is critical for a better sequencing strategy. Therefore, we investigated the amount of MinION data needed to assemble a high-quality genome by changing the total sequence reads from $C$. variabilis, such as $5 \times, 10 \times, 20 \times, 30 \times$, and $40 \times$ MinION read data. We then estimated the amount of MinION data required to establish a pipeline by evaluating these assemblies' varying read depths using indices such as the number of contigs, ECR, and RNAseq mapping rate. For assemblies using $5 \mathrm{X}$ data, the number of contigs, ECR, and RNA-seq mapping rate were $3809,78.9 \%$, and $80.87 \%$, respectively (Fig. 4a). Using 10X, the number of contigs was obviously reduced, whereas the ECR and RNA-seq mapping rate were similar to those for the MaSuRCA-assembly (Fig. 4a). For assemblies using 20X data, all indices were similar to those for the MaSuRCA-assembly
(Fig. 4a). A possible reason for the lack of improvement in the assembly indices after $10-20 \times$ is the sequence differences between the error-prone MinION raw reads and short reads. Given these results, we suggest that the high-quality genome is assembled using MinION read data of 10-20X the genome size.

Next, to reduce the number of contigs, we performed scaffolding MaSuRCA-assembly using Redundans [27], which reduces the redundant contigs and performs scaffolding. We examined the optimal conditions of Redundans by checking the assembly after each step of the Redundans pipeline using indices such as total length, number of contigs, ECR, and RNA-seq mapping rate. We found that the total length was about $10 \mathrm{Mbps}$ shorter than the reference assembly and that the ECR and RNA-seq mapping rates were drastically reduced after the scaffolding step using long reads (Fig. 4b). For Redundans without scaffolding using long reads, the number of contigs, the largest contig, N50, the reference coverage, and the percent sequence identity to the reference were 187, 2,552,940 bp, 611,131 bp, 99.32\%, and $95.58 \%$, respectively, demonstrating that the ECR and RNA-seq mapping rates were the same as the originals (Fig. 5a). A possible reason for the reduction in assembly

a

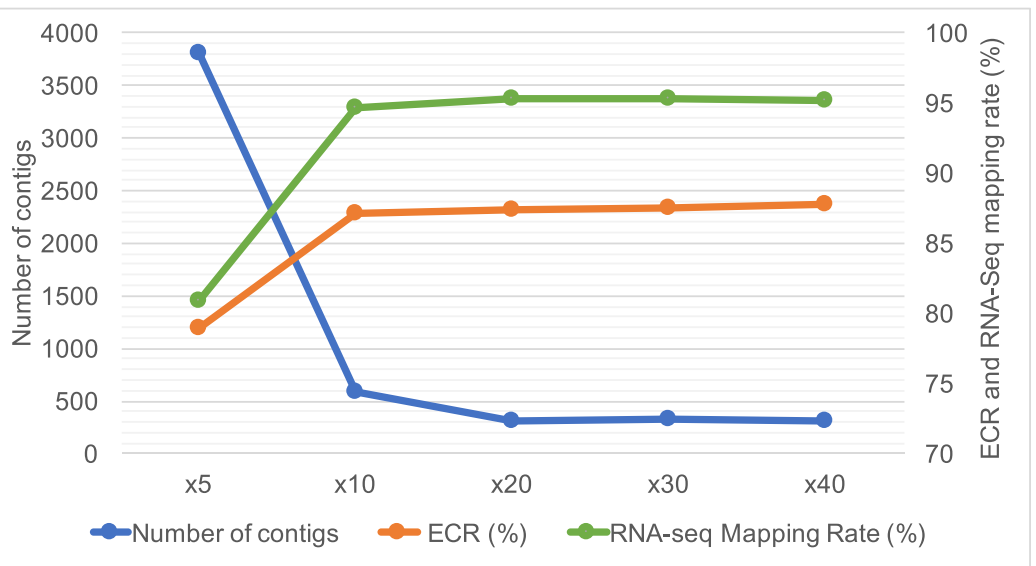

b

\begin{tabular}{|c|c|c|c|c|}
\hline Step of Redundans & Total length (bp) & Number of contigs & ECR (\%) & RNA-Seq mapping rate (\%) \\
\hline Start & $46,674,734$ & 302 & 88.5 & 95.06 \\
\hline Reduction & $46,017,283$ & 197 & 88.5 & 95.09 \\
\hline $\begin{array}{c}\text { Scaffolding using short-reads } \\
\text { Scaffolding using long-reads }\end{array}$ & $46,017,283$ & 190 & 88.5 & 95.08 \\
\hline Gapclosing & $37,162,854$ & 128 & 71.9 & 76.22 \\
\hline Reduction & $37,162,854$ & 128 & 71.9 & 76.22 \\
\hline
\end{tabular}

Fig. 4 Optimization of an assembly pipeline by changing genome coverage and by reducing contigs. a Evaluation of assemblies varing read depth. The blue line shows number of contigs, the orange line shows ECR, and the green line shows RNA-seq mapping rate. The $x$-axis indicates read depth. $\mathbf{b}$ Examination of optimal conditions of Redundans for improving MaSuRCA-assemblies. The first column represents each step of Redundans pipeline. Each column represents index for evaluating these assemblies 
indices after scaffolding with long reads is sequence differences between the error-prone MinION raw reads and the MaSuRCA-assembly corrected by the short reads. In summary, we adopted Redundans without a scaffolding step using long read to brush up the pipeline, as this resulted in a reduced number of contigs.

The next step was gene prediction from the assembly using BRAKER1 [28], a pipeline for unsupervised RNAseq-based gene prediction. Using this method, we found 13,008 genes (Fig. 5a). The ECR of this gene model was about 10\% higher than that of the gene model (reference), indicating that this gene model was more precise (Fig. 5a). To assess the comprehensiveness of this gene model, we examined how much RNA-seq could be mapped to the reference and current gene models. The RNA-seq mapping rates to the gene model (reference) and predicted gene model were $56.32 \%$ and $62.66 \%$, respectively (Fig. 5a). This result indicates that the RNA-seq mapping rate was more precise than the gene model (reference) for predicting the gene model. However, we also found unmapped RNA-seq reads to the current gene model, as there was a discrepancy of about $30 \%$ between the RNA-seq mapping rate to the gene model using BRAKER1 and mapping rate to the assembled genome. We hypothesized that the unmapped reads might be derived from transcripts from predicted gene-related regions, such as introns, and the upstream and downstream regions of this gene model. We then aligned the RNA-seq reads to the region related to the gene model and found that $3.92 \%$ of RNA-seq reads mapped to the intron region and $22.50 \%$ mapped to the upstream and downstream regions (Fig. 6). These results supported our hypothesis, as $89.08 \%$ of RNA-seq reads were mapped to this gene model, including the intron and upstream and downstream regions. These results also indicate that untranslated regions might be predicted using RNA-seq. Moreover, we found that $6 \%$ of RNA-seq reads could not be mapped to the gene model as other transcripts. To investigate what these reads were, we constructed de novo RNA assembly annotated against Arabidopsis thaliana by means of a BLAST search. From the gene set enrichment analysis (GSEA) using gene ontology (GO) of annotated contigs, the following $\mathrm{GO}$ terms were enriched: in the biological process (BP), oxidation-reduction process (GO:0055114) and photosynthesis (GO:0015979); in the cell component

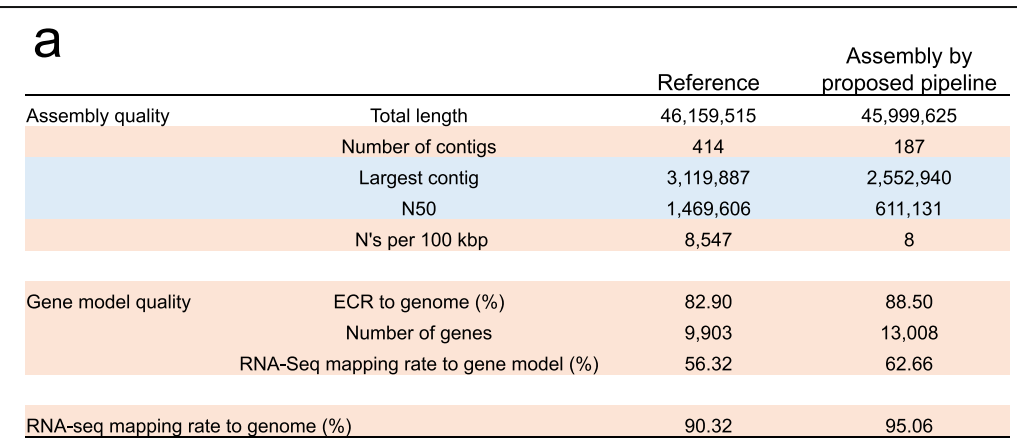

b

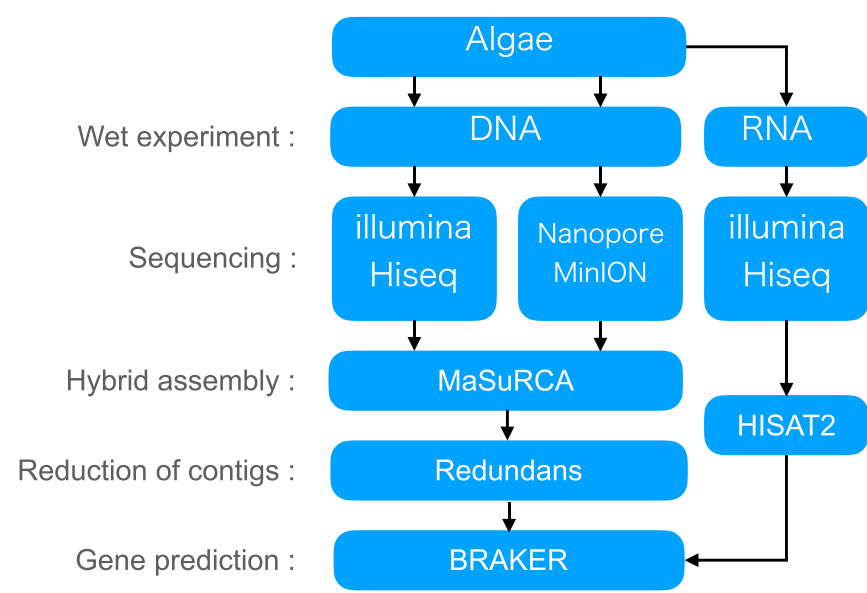

Fig. 5 Overview of this study. a Comparison between reference (Reference) and most high-quality assembly (Assembly) in this study. Assebly by proposed pipeline generated by hybrid assembly using MaSuRCA, and then brushing up by Redundans. Improved indexes compared to reference are red colored, in contrast indexes which did not improved compared to reference are blue. $\mathbf{b}$ Overview of constructed pipeline of de novo assembly for middle-sized genome using MinION 


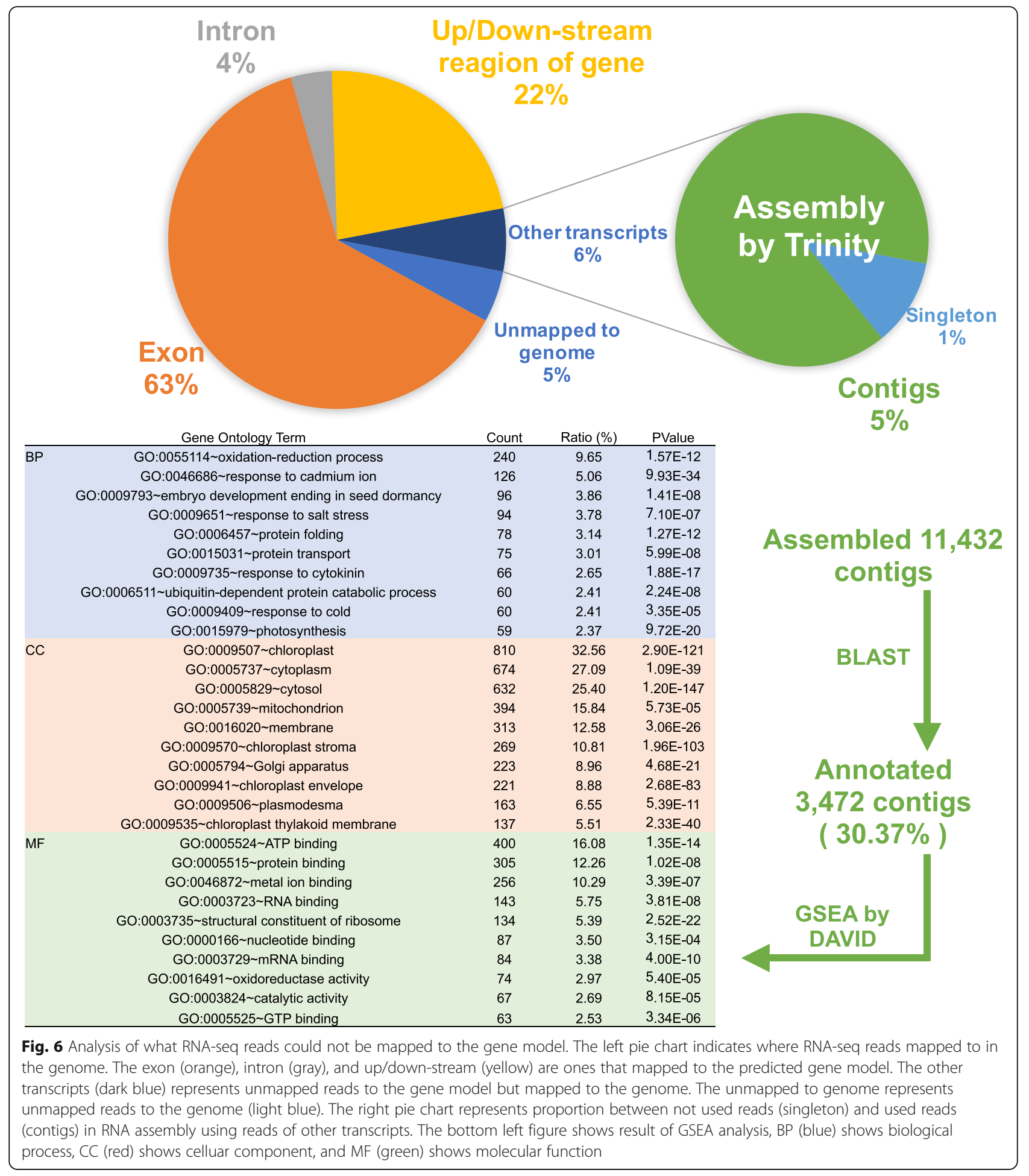

(CC), chloroplast (GO:0009507), and mitochondrion (GO:0005739), etc. (Fig. 6). These results suggest that genes coded in the genome of mitochondria and chloroplasts might be not precisely predicted because BRAKER1 software predicts eukaryote genes, and these are derived from prokaryotes [28].
For the pipeline of de novo assembly of middle-sized genomes using MinION, a high-quality genome could be assembled by MaSuRCA using 80X coverage of short reads and 10-20X MinION reads. This assembly could be brushed up by Redundans without the scaffolding step using long read to reduce contigs. Finally, the genes 
were precisely predicted by BRAKER1 using RNA-seq (Fig. 5b). For the genome of C. variabilis assembled using this pipeline, the number of contigs and $\mathrm{N}$ were lower, but the largest contig and N50 were shorter than those for the reference using Sanger sequences with BAC clones (Fig. 5a). The number of contigs over 50 $\mathrm{kbp}$ was larger than that in the reference, indicating that each contig was longer on average. Our assembly is useful for analysis of horizontal gene transfer and synteny (Additional file 1: Figure S2).

\section{Conclusions}

In this study, we sequenced $C$. variabilis using MinION and assembled genomes under various conditions. We then evaluated these assemblies in detail using assembly quality and alignment to reference as well as gene model quality and RNA-seq mapping rate. Evaluation of these assemblies revealed that the assembly with MinION reads only was not useful for comparative genomics and transcriptomics, as the percent sequence identity to the reference, gene prediction accuracy, and RNA-seq mapping rate were low. The cause of these low values was the low sequencing accuracy of MinION. However, this problem was resolved by hybrid assembly using the MaSuRCA assembler, and we then assembled a highquality genome at levels comparable with the reference assembly and predicted a more accurate gene model. By this assembly, we established the pipeline of de novo assembly for middle-sized genome using MinION. The genomic change during the early stages of plastid acquisition can now be revealed by sequencing and comparing many algal genomes. Moreover, this pipeline offers a solution for the assembly of various middle-sized eukaryotic genomes with high-quality and ease.

\section{Methods}

\section{DNA \& RNA preparation}

Chlorella variabilis strain NC64A (ATCC 50258) was cultured under illumination by a light-emitting diode lamp (14 h/10 h light/dark cycle) at room temperature (around $25{ }^{\circ} \mathrm{C}$ ). We used 20\% Gamborg's B5 basal medium with mineral organics (Sigma-Aldrich, MO, USA) at $\mathrm{pH} 7.5$ (referred to as $1 / 5 \mathrm{G}$ medium), with $0.02 \%$ l-serine added (1/5 G+Serin) as the culture medium. Under these culture conditions, Chlorella cells in the stationary phase were collected by centrifugation for DNA and RNA extractions.

DNA extraction was performed using the DNeasy Plant Mini Kit (Qiagen, Düsseldorf, Germany) with modified cell fracturing. Fifty to one hundred $\mu$ g of cells were incubated for $5 \mathrm{~min}$ in $400 \mu \mathrm{L}$ Buffer AP1 with $4 \mu \mathrm{L}$ RNase A at $65{ }^{\circ} \mathrm{C}$. After adding $400 \mu \mathrm{L}$ of glass beads ( $\varnothing$ $0.1 \mathrm{~mm}$ ), each sample was homogenized in BeadSmash 12 (WakenBTech, Kyoto, Japan) at $5000 \mathrm{rpm}$ for $30 \mathrm{~s}$.
Homogenization was repeated five times, and then each sample was again incubated for $10 \mathrm{~min}$ at $65{ }^{\circ} \mathrm{C}$. Subsequent procedures were performed according to the manufacturer's directions.

For RNA extraction, the collected Chlorella cells were once reserved in 10-fold the volume of RNAlater Stabilization Solution (ThermoFisher, MA, USA). After adding $400 \mu \mathrm{L}$ of glass beads to each $400 \mu \mathrm{L}$ solution, homogenization was performed as in the method for DNA extraction. We then added $500 \mu \mathrm{L}$ of Buffer RLT (RNeasy Mini Kit, Qiagen) and vortexed and incubated the samples at $56{ }^{\circ} \mathrm{C}$ for $3 \mathrm{~min}$. After centrifugation at $13,000 \mathrm{~g}$, the supernatant was transferred to a new tube. Subsequent procedures were performed according to the directions of the RNeasy Mini Kit.

\section{Illumina library preparation and sequencing}

Extracted genomic DNA and total RNA of C. variabilis were shipped to BGI-Shenzhen (Shenzhen, China) for library preparation and sequencing. For the DNA-seq and RNA-seq datasets, we sequenced genomic DNA and total RNA of C. variabilis using HiSeq 4000 and obtained $2 \times 100$ bp 35,007,584 and 25,396,225 paired-end reads, respectively.

\section{MinION library preparation and sequencing}

Genomic DNA $(6.4 \mu \mathrm{g})$ was fragmented to approximately $8 \mathrm{kbp}$ using Covaris g-TUBE (Covaris, Woburn, USA). After purification using $0.4 \times$ AMPure XP beads (Beckman Coulter Inc., Brea, CA, USA) without a DNA repair step, the sequencing library was prepared using the SQK-LSK108 sequencing kit (Oxford Nanopore Technologies, Oxford, UK) following the manufacturer's protocol (1D Genomic DNA by ligation). The sequencing library was loaded to R9 chemistry flowcell (FLO-MIN106) and sequenced with live base calling using MinKNOW for $48 \mathrm{~h}$. FASTA and FASTQ files and the sequencing summary were generated using Poretools [29].

\section{De novo genome assembly and evaluation}

The reference genome of C. variabilis (unmasked ChlNC64A_1_nuclear_scaffolds.fasta) was downloaded from the JGI Genome Portal [30]. After quality control using AfterQC [31], short reads were derived from HiSeq assembled by SPAdes v3.10.1 [14] with the default parameters. All MinION reads were assembled using ABrujin v1.0 [17] with parameters: -o 3000, Miniasm v.0.2 [18] pipeline with default parameters and Canu v1.5 [19] with default parameters. Hybrid assembly was performed using SPAdes v3.10.1 [14] with parameters: --nanopore and MaSuRCA v3.2.2 [20] with default parameters. The ABrujin-assembly was polished using Racon [21] and Nanopolish [22] using the base called data with Albacore v3.0.0 [32]. The indices of assembly 
quality were evaluated by Quast [33]. The reference coverage and percent sequence identity to the reference were calculated using Minimap2 v2.6 [34] with parameters: -ax asm5. The reference coverage was calculated from "the number of bases in mapped sequences" derived from Minimap2 [34] divided by the size of the reference genome. Similarly, the percent sequence identity to the reference was calculated from "the number of mapped bases" [34] divided by the size of the reference genome.

\section{Gene prediction accuracy and RNA-seq mapping rate}

To evaluate gene prediction accuracy, the ECR of the assembly was calculated as the ratio of core genes whose full lengths were expected, including duplicated core genes using BUSCO with Eucaryota odb9 datasets (303 single-copy orthologs) [23]. The reference gene model of C. variabilis was Chlorella_NC64A.best_genes.gff deposited in the JGI Genome Portal [30]. The gene model was predicted using the BRAKER1 [28] pipeline with the default settings and evaluated using indices of the number, average length, and ECR generated by an R script and BUSCO [23]. The reads of the RNA-seq were mapped to the assembled genome using HISAT2 [24] with default settings, and then TPM [25] was calculated using StringTie [35]. To obtain the same genes from the reference, short-read-assembly, ABrujin-polished-assembly, and MaSuRCA-assembly orthologs were predicted using Orthofinder [36] and the single-copy orthologs were extracted. The correlation coefficient and scatterplot of TPM of extracted orthologs were generated by Python and R scripts.

\section{Establishment of pipeline}

To examine MinION read depth for the hybrid assembly, genomes of C. variabilis were assembled using the MaSuRCA-assembler with $5 \times, 10 \times, 20 \times, 30 \times$, and $40 \times$ MinION data, respectively. Next, these assemblies were evaluated on the basis of the number of contigs, ECR, and RNA-seq mapping rate. The optimal conditions of Redundans were examined by assessing the total length, number of contigs, ECR, and RNA-seq mapping rate for each genome assembly after the first reduction, scaffolding with short reads, scaffolding with long reads, gap closing, and final reduction steps of the Redundans pipeline, respectively. To use BRAKER1, we evaluated the gene model predicted from the brushed up genome. RNA-seq was mapped to this gene model and the reference gene model. Next, the mapping rates were calculated and compared. Furthermore, to characterize the unmapped reads, RNA-seq data were mapped to the intron and the upstream and downstream regions of the predicted gene model. The mapping rate for the predicted gene-related region was also calculated. Lastly, the upstream and downstream region was extracted from peripheral $1 \mathrm{kbp}$ sequences for each predicted gene.

\section{Analysis of unmapped reads to the gene model}

Unmapped RNA-seq reads to the gene model were assembled using Trinity v2.4.0 [37], and assembled contigs were then annotated using BLASTp with an e-value threshold of $10^{-5}$ to the A. thaliana reference proteome data (UniProt Proteome ID: UP000006548). This blast result was analyzed by GSEA using DAVID [38] and the top 10 most frequent GO terms in the BP, CC, and MF category.

\section{Additional files}

Additional file 1: Figure S1. Histogram of the length of raw reads derived form MinION. Much short reads under 500 bp were removed by means of purification using $0.4 \times$ magnetic beads before library preparation. Figure S2. The contigs of assembled genomes of $C$. variabilis sorted in descending order of the length. Reference were previously assembled genome, assembly were assembled genome using the pipeline constructed in this study. (PDF $95 \mathrm{~kb}$ )

Additional file 2: Table S1. Summary of sequencing using MinION. (XLSX $9 \mathrm{~kb}$ )

\section{Abbreviations \\ Core gene: 303 eukaryotic common single-copy-orthologs; ECR: Eukaryotic common gene conservation rate; GO: Gene ontology; GSEA: Gene set enrichment analysis}

\section{Acknowledgements}

We appreciate the staff of Nagahama Institute of Bio-Science and Technology Genomic diversity laboratory, Ikuyo Takemura for assistance with experiment and analysis. $\mathrm{RH}$ and $\mathrm{AO}$ are financially aided by Japan Society for the Promotion of Science KAKENHI Grant Number 16 K07491, 17H06399, and Grant for Basic Science Research Projects from the Sumitomo Foundation.

\section{Funding}

This work was funded by Grants-in-Aid for Scientific Research (KAKENHI Grant Number 16K07491, and 17H06399) to $\mathrm{AO}, \mathrm{RH}$.

Availability of data and materials

All sequence data are deposited in DDBJ (accession number is DRA007289).

\section{Authors' contributions}

$\mathrm{AO}$ conceived the project, designed the content, and organized the manuscript. RM conducted the sequencing experiment using MinION, analyzed data, and drafted the manuscript. RH prepared genomic DNA and RNA of C. variabilis and contributed to drafting the manuscript. All authors read and approved the final manuscript.

Ethics approval and consent to participate Not applicable.

\section{Consent for publication}

Not applicable.

\section{Competing interests}

The authors declare that they have no potential conflict of interest. Dr. Ogura, associate editor for BMC genomics, was not involved in the editorial review of or decision to publish this article. 


\section{Publisher's Note}

Springer Nature remains neutral with regard to jurisdictional claims in published maps and institutional affiliations.

Received: 27 March 2018 Accepted: 11 September 2018

Published online: 24 September 2018

\section{References}

1. Delwiche CF. Tracing the thread of plastid diversity through the tapestry of life. Am Nat. 1999;154:S164-77.

2. McFadden Gl. Primary and secondary endosymbiosis and the origin of plastid. J Phycol. 2001;37:951-9.

3. Keeling PJ. Diversity and evolutionary history of plastids and their hosts. Am J Bot. 2004;91:1481-93.

4. Inouye I, Okamoto N. Changing concepts of a plant: current knowledge on plant diversity and evolution. Plant Biotechnol. 2005;22:505-14.

5. Curtis BA, Tanifuji G, Burki F, Gruber A, Irimia M, Maruyama S, et al. Algal genomes reveal evolutionary mosaicism and the fate of nucleomorphs. Nature. 2012;492:59-65.

6. Hoshina R, Iwataki M, Imamura N. Chlorella variabilis and Micractinium reisseri sp. nov. (Chlorellaceae, Trebouxiophyceae): Redescription of the endosymbiotic green algae of Paramecium bursaria (Peniculia, Oligohymenophorea) in the 120th year: taxonomy of the photobionts of Paramecium. Phycol Res. 2010:58:188-201.

7. Blanc G, Duncan G, Agarkova I, Borodovsky M, Gurnon J, Kuo A, et al. The Chlorella variabilis NC64A genome reveals adaptation to photosymbiosis, coevolution with viruses, and cryptic sex. Plant Cell. 2010;22:2943-55.

8. Hoshina R, Kusuoka Y. DNA analysis of algal endosymbionts of ciliates reveals the state of algal integration and the surprising specificity of the Symbiosis. Protist. 2016;167:174-84.

9. Orsini M, Cusano R, Costelli C, Malavasi V, Concas A, Angius A, et al. Complete genome sequence of chloroplast DNA (cpDNA) of Chlorella sorokiniana. Mitochondrial DNA. 2016:27:838-9.

10. Gao C, Wang Y, Shen Y, Yan D, He X, Dai J, et al. Oil accumulation mechanisms of the oleaginous microalga Chlorella protothecoides revealed through its genome, transcriptomes, and proteomes. BMC Genomics. 2014; 15:582.

11. Ota S, Oshima K, Yamazaki T, Kim S, Yu Z, Yoshihara M, et al. Highly efficient lipid production in the green alga Parachlorella kessleri: draft genome and transcriptome endorsed by whole-cell 3D ultrastructure. Biotechnol Biofuels. 2016;9.1:13.

12. Nanoporetech.com Learn More. 2009:99. https://nanoporetech.com/learnmore. Accessed 9 Jan 2018.

13. Pacific Biosciences - AllSeq. http://allseq.com/knowledge-bank/sequencingplatforms/pacific-biosciences/. Accessed 9 Jan 2018.

14. Lu H, Giordano F, Ning Z. Oxford Nanopore MinION sequencing and genome assembly. Genomics Proteomics Bioinformatics. 2016;14:265-79.

15. Giordano F, Aigrain L, Quail MA, Coupland P, Bonfield JK, Davies RM, et al. De novo yeast genome assemblies from MinION, PacBio and MiSeq platforms. Sci Rep. 2017;7:3935.

16. Bankevich A, Nurk S, Antipov D, Gurevich AA, Dvorkin M, Kulikov AS, et al. SPAdes: a new genome assembly algorithm and its applications to singlecell sequencing. J Comput Biol. 2012;19:455-77.

17. Lin Y, Yuan J, Kolmogorov M, Shen MW, Chaisson M, Pevzner PA. Assembly of long error-prone reads using de Bruijn graphs. Proc Natl Acad Sci U S A. 2016;113:E8396-405.

18. Li H. Minimap and miniasm: fast mapping and de novo assembly for noisy long sequences. Bioinformatics. 2016;32:2103-10.

19. Koren S, Walenz BP, Berlin K, Miller JR, Bergman NH, Phillippy AM. Canu: scalable and accurate long-read assembly via adaptive k-mer weighting and repeat separation. Genome Res. 2017:27:722-36.

20. Zimin AV, Marçais G, Puiu D, Roberts M, Salzberg SL, Yorke JA. The MaSuRCA genome assembler. Bioinformatics. 2013;29:2669-77.

21. Vaser R, Sović I, Nagarajan N, Šikić M. Fast and accurate de novo genome assembly from long uncorrected reads. Genome Res. 2017:27:737-46.

22. Jared Simpson Github repository. https://github.com/jts/nanopolish. Accessed 9 Jan.

23. Simão FA, Waterhouse RM, loannidis P, Kriventseva EV, Zdobnov EM. BUSCO: assessing genome assembly and annotation completeness with single-copy orthologs. Bioinformatics. 2015;31:3210-2.
24. Kim D, Langmead B, Salzberg SL. HISAT: a fast spliced aligner with low memory requirements. Nat Methods. 2015:12:357-60.

25. Wagner GP, Kin K, Lynch VJ. Measurement of mRNA abundance using RNA-seq data: RPKM measure is inconsistent among samples. Theory Biosci. 2012;131:281-5

26. Kamako S, Hoshina R, Ueno S, Imamura N. Establishment of axenic endosymbiotic strains of Japanese Paramecium bursaria and the utilization of carbohydrate and nitrogen compounds by the isolated algae. Eur J Protistol. 2005:41:193-202.

27. Pryszcz LP, Gabaldón T. Redundans: an assembly pipeline for highly heterozygous genomes. Nucleic Acids Res. 2016:44:e113.

28. Hoff KJ, Lange S, Lomsadze A, Borodovsky M, Stanke M. BRAKER1: unsupervised RNA-Seq-based genome annotation with GeneMark-ET and AUGUSTUS. Bioinformatics. 2016;32:767-9.

29. Loman N, Quinlan A. Poretools: a toolkit for analyzing nanopore sequence data. Bioinformatics. 2014;30.23:3399-3401.

30. JGl Genome Portal - Chlorella sp. NC64A. https://genome.jgi.doe.gov/ ChINC64A_1/ChINC64A_1.home.html. Accessed 9 Jan 2018.

31. Chen S, Huang T, Zhou Y, Han Y, Xu M, Gu J. AfterQC: automatic filtering, trimming, error removing and quality control for fasta data. BMC Bioinformatics. 2017;18(Suppl 3):80.

32. Albacore Github repository. https://github.com/Albacore/albacore. Accessed 9 Jan

33. Gurevich A, Saveliev V, Vyahhi N, Tesler G. QUAST: quality assessment tool for genome assemblies. Bioinformatics. 2013;29:1072-5.

34. Li H. Minimap2: versatile pairwise alignment for nucleotide sequences. 2017. http://arxiv.org/abs/1708.01492. Accessed 9 Jan 2018.

35. Pertea M, Kim D, Pertea GM, Leek JT, Salzberg SL. Transcript-level expression analysis of RNA-seq experiments with HISAT, StringTie and Ballgown. Nat Protoc. 2016;11:1650-67.

36. Emms DM, Kelly S. OrthoFinder: solving fundamental biases in whole genome comparisons dramatically improves orthogroup inference accuracy. Genome Biol. 2015:16:157.

37. Haas BJ, Papanicolaou A, Yassour M, Grabherr M, Blood PD, Bowden J, et al. De novo transcript sequence reconstruction from RNA-seq using the trinity platform for reference generation and analysis. Nat Protoc. 2013:8:1494-512.

38. Huang DW, Sherman BT, Lempicki RA. Systematic and integrative analysis of large gene lists using DAVID bioinformatics resources. Nat Protoc. 2009:4:44-57.

Ready to submit your research? Choose BMC and benefit from:

- fast, convenient online submission

- thorough peer review by experienced researchers in your field

- rapid publication on acceptance

- support for research data, including large and complex data types

- gold Open Access which fosters wider collaboration and increased citations

- maximum visibility for your research: over $100 \mathrm{M}$ website views per year

At BMC, research is always in progress.

Learn more biomedcentral.com/submissions 